\title{
One-pot synthesis of triptycene-based porous organic frameworks with tailored micropore environments for highly efficient and selective amine adsorption
}

\author{
Haijiang Wang, Lili Pan, Wenxiu Deng, Guangjie Yang and Xikui Liu \\ Triptycene-based porous organic frameworks (POFs) with high BET (Brunauer-Emmett-Teller) surface areas and tailored \\ micropore environments were prepared using a facile external knitting strategy. The functionalized POFs exhibited very high \\ aliphatic amine vapor uptakes of up to 5 times their own weight and more than $\mathbf{3 0}$ times selectivity for aliphatic amines over \\ $n$-hexane; thus, they provide new opportunities for environment-related applications. \\ Polymer Journal (2016) 48, 787-792; doi:10.1038/pj.2016.20; published online 2 March 2016
}

\section{INTRODUCTION}

Volatile organic compounds (VOCs) such as benzene, toluene, alkanes and amines can readily evaporate into the atmosphere and become hazardous air pollutants. ${ }^{1-3}$ The environmental concerns associated with these compounds create an urgent need for new functional materials for the elimination of air pollutants. ${ }^{4-7}$ Among them, porous organic frameworks (POFs) have attracted great attention because of their high surface area and good chemical stability. ${ }^{8-13}$ For example, Jiang and colleagues ${ }^{14}$ synthesized various kinds of porphyrin microporous polymers and reported their efficiency in the uptake of amines. In addition to the high BET (Brunauer-Emmett-Teller) surface area, the pore functionalization is also very important to achieve highly efficient adsorption.

Iptycene derivatives have attracted significant attention in supramolecular chemistry in the past decade. ${ }^{15}$ Their rigid frameworks with constrained aromatic groups between the bridgehead carbons at an angle close to $120^{\circ}$ lead to void spaces called the 'internal molecular free volume.' This void space has been used to introduce intrinsic microporosity and host-guest interactions into materials, including polymers and metal-organic frameworks. ${ }^{16,17}$ Several triptycene-based microporous polymers have also been synthesized from the palladium-catalyzed coupling polymerization. However, scale up may be challenging because the monomers, such as triiodo- or hexahydroxy-triptycene, and the palladium catalyst are quite expensive. ${ }^{18,19}$

In recent years, a versatile external knitting strategy based on Friedel-Craft reaction was demonstrated for the facile synthesis of microporous polymers. ${ }^{20,21}$ Based on this strategy, we designed and synthesized three triptycene-based porous organic frameworks (TPOFs) using triptycene hydroquinone and glyoxylic acid as the knitting agent. Further study showed that the functionalized POFs exhibit high aliphatic amine vapor uptakes of up to 5 times of their own weight, higher than that of any microporous materials reported so far. In addition, they show high selectivity of more than 30 times for aliphatic amines over non-polar $n$-hexane. This extremely high vapor uptake and selectivity should greatly benefit their use in environment-related applications Scheme 1.

\section{EXPERIMENTAL PROCEDURE}

\section{Materials}

Triptycene and triptycene-1,4-hydroquinone were prepared and purified according to standard procedures. 1,2-Dichloroethane was purchased from Chengdu Kelong Chemical Regent Co. (Chengdu, China) and purified by distillation under reduced pressure and dried with $4 \mathrm{~A}$ molecular sieves. Other chemicals were purchased from Chengdu Kelong Chemical Regent Co. and purified by standard methods.

\section{Synthesis of TPOFs}

Three kinds of TPOFs were synthesized via a one-step solution polymerization method using paraformaldehyde as the external crosslinker. A typical polymerization procedure for TPOF is as follows: a mixture of triptycene $(516 \mathrm{mg})$ and paraformaldehyde $(360 \mathrm{mg}$ ) was dissolved in anhydrous 1,2-dichloroethane $(12 \mathrm{ml})$ containing $2 \mathrm{~g}$ of anhydrous $\mathrm{FeCl}_{3}$. The solution was sonicated for $15 \mathrm{~min}$ at room temperature. It was then put in a Teflon-lined autoclave and transferred into an oven and heated at $45^{\circ} \mathrm{C}$ for $5 \mathrm{~h}$, followed by $120^{\circ} \mathrm{C}$ for $48 \mathrm{~h}$ to finish the polycondensation. The precipitate was collected by filtration and washed with hydrochloric acid and 1,2-dichloroethane. The resultant TPOF was purified in a Soxhlet apparatus with methanol for $24 \mathrm{~h}$ and dried at $120^{\circ} \mathrm{C}$ under vacuum for $12 \mathrm{~h}$. TPOF was obtained in $96 \%$ isolated yield as a brown solid.

The trypticene-based porous organic framework with hydroxyl groups (TPOF-H) was prepared using a similar procedure except that the triptycene hydroquinone $(580 \mathrm{mg})$ was condensated with paraformaldehyde $(360 \mathrm{mg})$. TPOF-H was obtained in 95\% isolated yield as a light brown solid.

The trypticene-based porous organic framework with hydroxyl and carboxyl groups (TPOF-HC) was prepared using a similar procedure except that the 


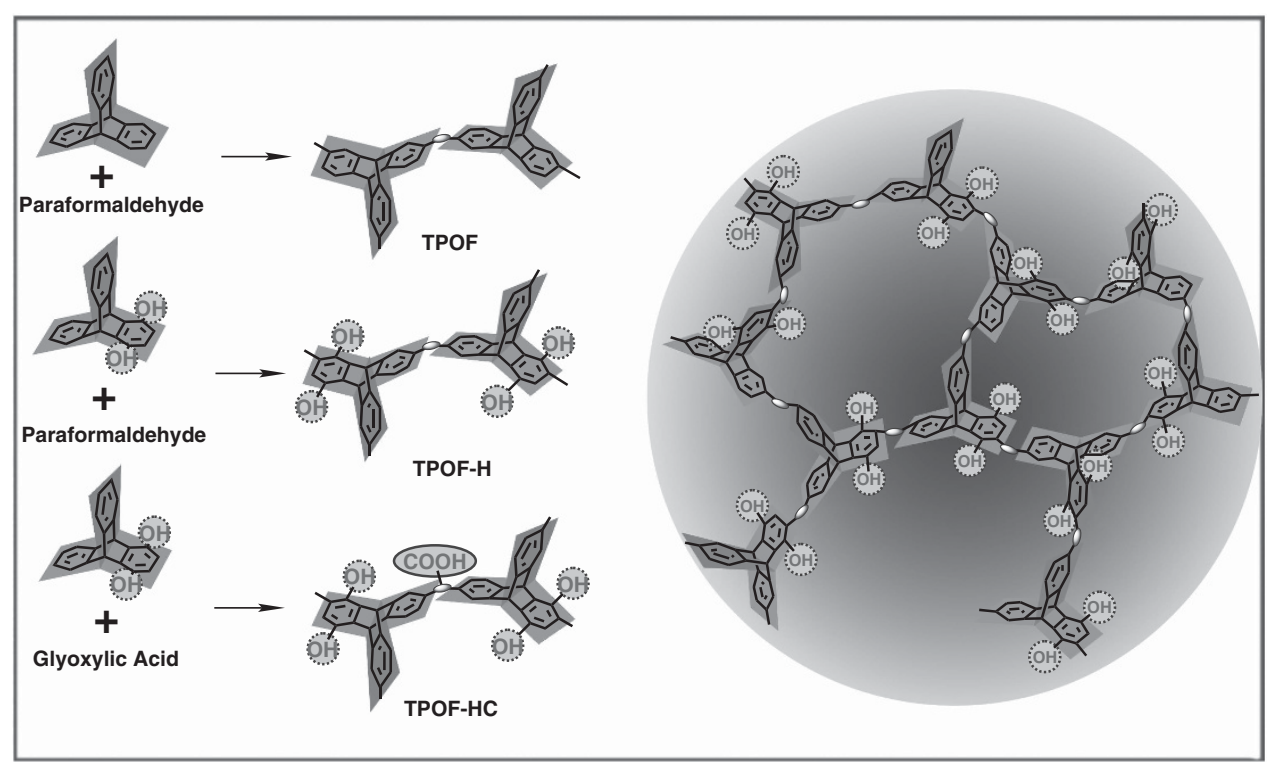

Scheme 1 Synthesis of triptycene-based porous organic frameworks: TPOF, TPOF-H and TPOF-HC. TPOF, triptycene-based porous organic framework; TPOF$\mathrm{H}$, TPOF with hydroxyl groups; TPOF-HC, TPOF with hydroxyl and carboxyl groups. A full color version of this figure is available at Polymer Journal online.

triptycene hydroquinone $(580 \mathrm{mg})$ was condensated with glyoxylic acid (414 mg). TPOF-HC was obtained in $91 \%$ isolated yield as a light brown solid.

\section{Characterizations}

Fourier transform infrared spectra were measured with a Nicolet 560 spectrometer (Waltham, MA, USA). Solid-state carbon-13 nuclear magnetic resonance spectra were recorded on a Bruker Avance III $500 \mathrm{MHz}$ with a standard 4-mm Bruker MAS Probe (Brugg, Switzerland) at a sample spinning rate of $8.0 \mathrm{kHz}$. Scanning electron microscopy was conducted with an Inspect F scanning electron microscope (FEI Company, Akishima-shi, Japan) at an accelerating voltage of $20 \mathrm{kV}$. The nitrogen absorption and desorption isotherms were measured at $77 \mathrm{~K}$ using a Tristar system. The sample was degassed at $150{ }^{\circ} \mathrm{C}$ for $10 \mathrm{~h}$ before the measurement. The surface area was calculated from the adsorption data using the BET method. The pore width distribution curves were obtained from the adsorption branch using the non-local density functional theory method. The thermal properties were measured using the thermogravimetric analysis instrument (SDT Q600, TA Instrument, New Castle, DE, USA) over the temperature range of 30 to $800{ }^{\circ} \mathrm{C}$ with a heating rate of $10^{\circ} \mathrm{C} \mathrm{min}^{-1}$.

\section{Vapor adsorption of VOCs}

All vapor absorption capacities were evaluated at $30^{\circ} \mathrm{C}$ at 1 bar. First, a weighted quantity of TPOF was placed in a small vial that was allowed to stand in a bottle with $1 \mathrm{ml}$ VOCs. Then, the bottle was sealed with a cap and left at $30^{\circ} \mathrm{C}$ for $240 \mathrm{~h}$. The increase in weight was used to evaluate the absorption capacity according to the following formula:

$$
\operatorname{Absorption} \operatorname{Capacity}(\mathrm{wt})=\left(W_{\mathrm{ti}}-W_{\mathrm{t} 0}\right) / \mathrm{W}_{\mathrm{dry}}
$$

where $W_{\mathrm{t} 0}$ and $W_{\mathrm{ti}}$ are the weight of the original sample and the weight after VOC adsorption for a certain time, respectively.

$$
\text { Absorption Selectivity }=W_{\mathrm{A}} / \mathrm{W}_{\mathrm{B}}
$$

where $W_{\mathrm{A}}$ and $W_{\mathrm{B}}$ are the absorption capacity of the POF for different kinds of VOCs.

\section{RESULTS AND DISCUSSION}

As shown in Scheme 1, triptycene was reacted with paraformaldehyde to obtain controlled POF (TPOF) as brown powders in good yield.
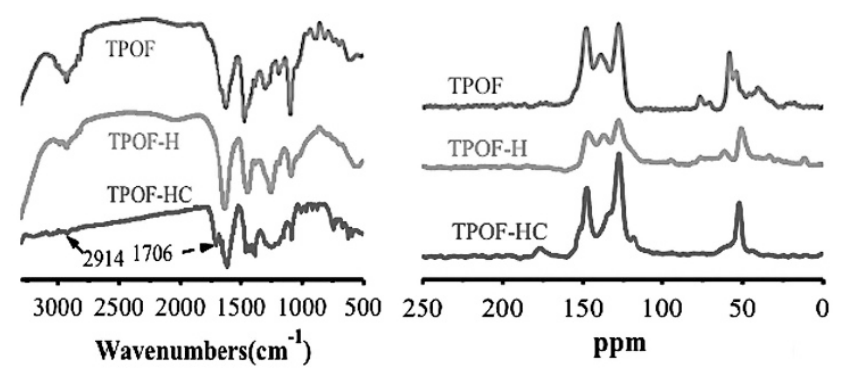

Figure 1 FT-IR and ${ }^{13} \mathrm{C}$ MAS-NMR spectra of TPOF, TPOF-H and TPOF-HC. FT-IR, Fourier transform infrared spectroscopy; ${ }^{13} \mathrm{C}$ MAS-NMR, carbon-13 magic-angle spinning-nuclear magnetic resonance; TPOF, triptycene-based porous organic framework; TPOF-H, TPOF with hydroxyl groups; TPOF-HC, TPOF with hydroxyl and carboxyl groups. A full color version of this figure is available at the Polymer Journal online.

To tailor the pore environment, triptycene-1,4-hydroquinone was reacted with paraformaldehyde to obtain TPOF-H, and triptycene-1,4-hydroquinone was reacted with glyoxylic acid to obtain TPOF-HC. The successful syntheses of POFs were confirmed by Fourier transform infrared spectroscopy and ${ }^{13} \mathrm{C}$-nuclear magnetic resonance (Figure 1). All of the POFs exhibit absorption peaks at 1600 , 1460 and $1080 \mathrm{~cm}^{-1}$, which resulted from the benzene frameworks, and peaks at $\sim 2850 \mathrm{~cm}^{-1}$, which resulted from the bridging methylene or methanetriyl groups. In addition, TPOF-HC showed an absorption at $1706 \mathrm{~cm}^{-1}$ that resulted from the carboxyl group. In the ${ }^{13} \mathrm{C}$-nuclear magnetic resonance spectra, the resonance peaks at 145 and 128 p.p.m. can be assigned to the aromatic carbons, and the resonance peaks at 55 p.p.m. can be assigned to the bridging methanetriyl and methylene carbons. In the case of TPOF-HC, the characteristic peak of the carbonyl carbon can be clearly identified at 175 p.p.m.

The morphologies of the resulting triptycene-based POFs were observed by field emission scanning electron microscopy. TPOF was composed mainly of loosely agglomerated nanoparticles, whereas 

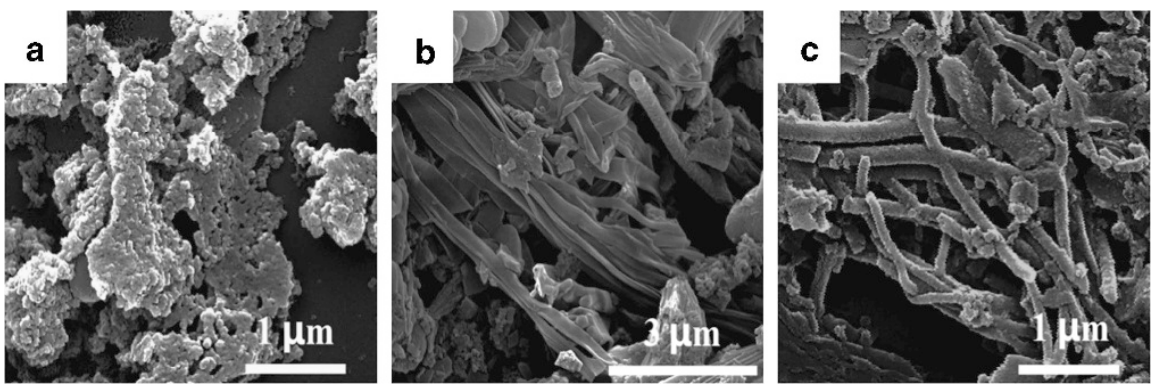

Figure 2 Scanning electron microscopy (SEM) images of TPOF (a), TPOF-H (b) and TPOF-HC (c). TPOF, triptycene-based porous organic framework; TPOF-H, TPOF with hydroxyl groups; TPOF-HC, TPOF with hydroxyl and carboxyl groups.
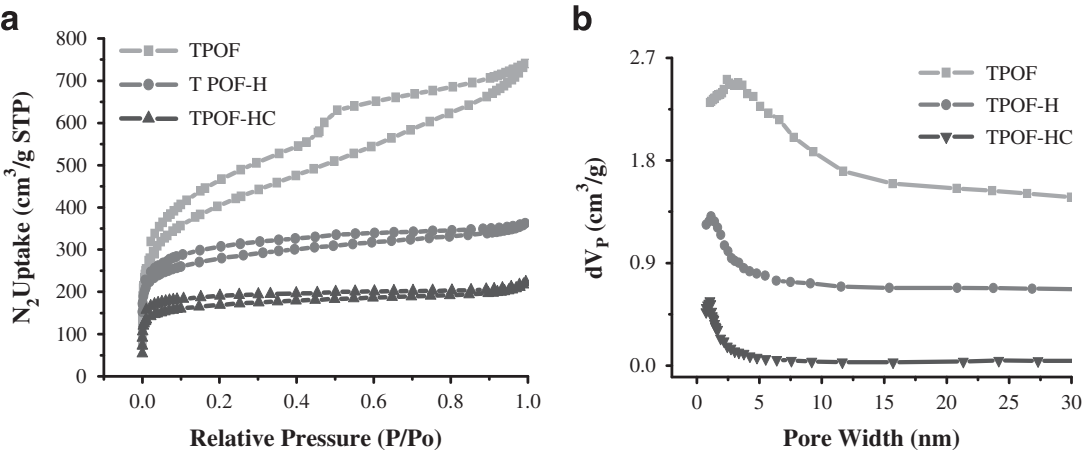

Figure 3 (a) Nitrogen adsorption and desorption isotherms measured at $77 \mathrm{~K}$ and (b) pore size distribution curves based on the density functional theory (DFT) model of the nitrogen adsorption isotherms of TPOF, TPOF-H and TPOF-HC. TPOF, triptycene-based porous organic framework; TPOF-H, TPOF with hydroxyl groups; TPOF-HC, TPOF with hydroxyl and carboxyl groups. A full color version of this figure is available at the Polymer Journal online.

TPOF-H and TPOF-HC were composed mainly of nanofibrous structures (Figure 2).

The permanent porosities of the TPOFs were determined by nitrogen sorption measurements at $77 \mathrm{~K}$. The sorption isotherms and pore distribution curves are shown in Figure 3. All of the isotherms exhibit steep rises at low relative pressures $\left(P / P_{0}<0.01\right)$ indicating their microporous nature. TPOF showed a pronounced hysteretic step in the $\mathrm{N}_{2}$ isotherm desorption, whereas there is no considerable hysteretic step for TPOF-H and TPOF-HC. This result was consistent with the pore size distribution curves. TPOF possess a rather broad pore geometry distribution from microporosity to mesoporosity and macroporosity, whereas TPOF-H and TPOF-HC showed mainly microporous structures with narrow pore size distributions.

The BET surface properties of these POFs are summarized in Table 1. The BET surface areas of TPOF, TPOF-H and TPOF-HC were 1444,1034 and $636 \mathrm{~m}^{2} \mathrm{~g}^{-1}$, respectively. The low value of TPOF-HC should be because of the low reactivity of glyoxylic acid compared with that of paraformaldehyde. However, even the total BET surface area is relatively low. TPOF-H has a microporosity BET surface area of $844 \mathrm{~m}^{2} \mathrm{~g}^{-1}$, higher than that of TPOF. Such a uniform microporosity and tailored pore environment should facilitate their application in size-selective adsorption.

The vapor adsorption of these triptycene-based POFs for various toxic VOCs were tested and are shown in Figure 4. The adsorption amounts of TPOF for aniline, benzene, triethylamine and $n$-hexane are $0.60,1.34,1.37$ and $1.35 \mathrm{~g} \mathrm{~g}^{-1}$, respectively; for $\mathrm{TPOF}-\mathrm{H}$, the adsorption amounts are $0.50,0.39,0.41$ and 0.20 , respectively; and for TPOF-HC, the adsorption amounts are $0.35,0.39,0.44$ and 0.14 ,
Table 1 Porosity parameters of TPOF, TPOF-H and TPOF-HC gained from nitrogen adsorption isotherms

\begin{tabular}{lccc}
\hline Samples & $S_{B E T}\left(m^{2} g^{-1}\right)$ & $S_{\text {micro }}\left(m^{2} g^{-1}\right)$ & $V_{\text {total }}\left(\mathrm{cm}^{3} \mathrm{~g}^{-1}\right)$ \\
\hline TPOF & 1444 & 701 & 0.93 \\
TPOF-H & 1034 & 844 & 0.49 \\
TPOF-HC & 636 & 553 & 0.26 \\
\hline
\end{tabular}

Abbreviations: TPOF, triptycene-based porous organic framework; TPOF-H, TPOF with hydroxyl groups; TPOF-HC, TPOF with hydroxyl and carboxyl groups.

respectively. The TPOFs with higher BET surface area showed higher adsorption capacities.

However, the situation was totally different when we further studied the vapor uptakes for various aliphatic amines (Figure 5). The functionalized TPOF-H and TPOF-HC showed much higher vapor adsorption capacities than TPOF. For TPOF-HC, even though it has a relatively low BET surface area of $636 \mathrm{~m}^{2} \mathrm{~g}^{-1}$, the vapor uptakes for cyclohexane, $n$-hexylamine, $n$-butylamine and $n$-propylamine are as high as $1.60,4.40,4.87$ and $4.58 \mathrm{gg}^{-1}$, respectively, whereas for TPOF-H, the values are even as high as 2.57, 4.36, 5.34 and $5.36 \mathrm{~g} \mathrm{~g}^{-1}$, respectively, thus revealing the extremely high adsorption capabilities of functionalized TPOF-H and TPOF-HC for aliphatic amines.

In addition to high adsorption capacities, TPOF-H and TPOF-HC also show high selectivities in VOC adsorption. As can be clearly identified in Figure 6, the adsorption selectivity of TPOF for aliphatic amines over aniline, benzene or triethylamine is generally $\sim 2$, whereas for TPOF-H and TPOF-HC, the selectivity is above 10. It is even high 

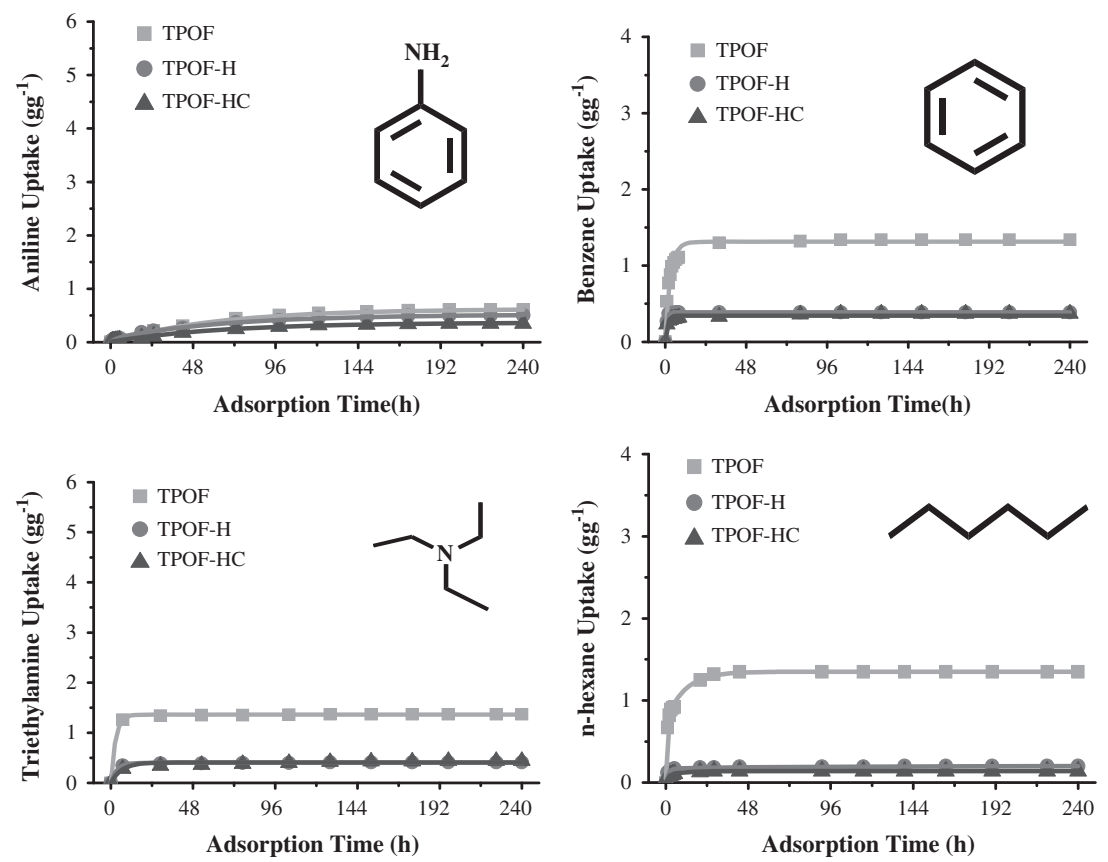

Figure 4 Vapor uptakes of aniline, pyridine, benzene, triethylamine, ammonia and $n$-hexane by TPOF, TPOF-H and TPOF-HC. TPOF, triptycene-based porous organic framework; TPOF-H, TPOF with hydroxyl groups; TPOF-HC, TPOF with hydroxyl and carboxyl groups. A full color version of this figure is available at the Polymer Journal online.
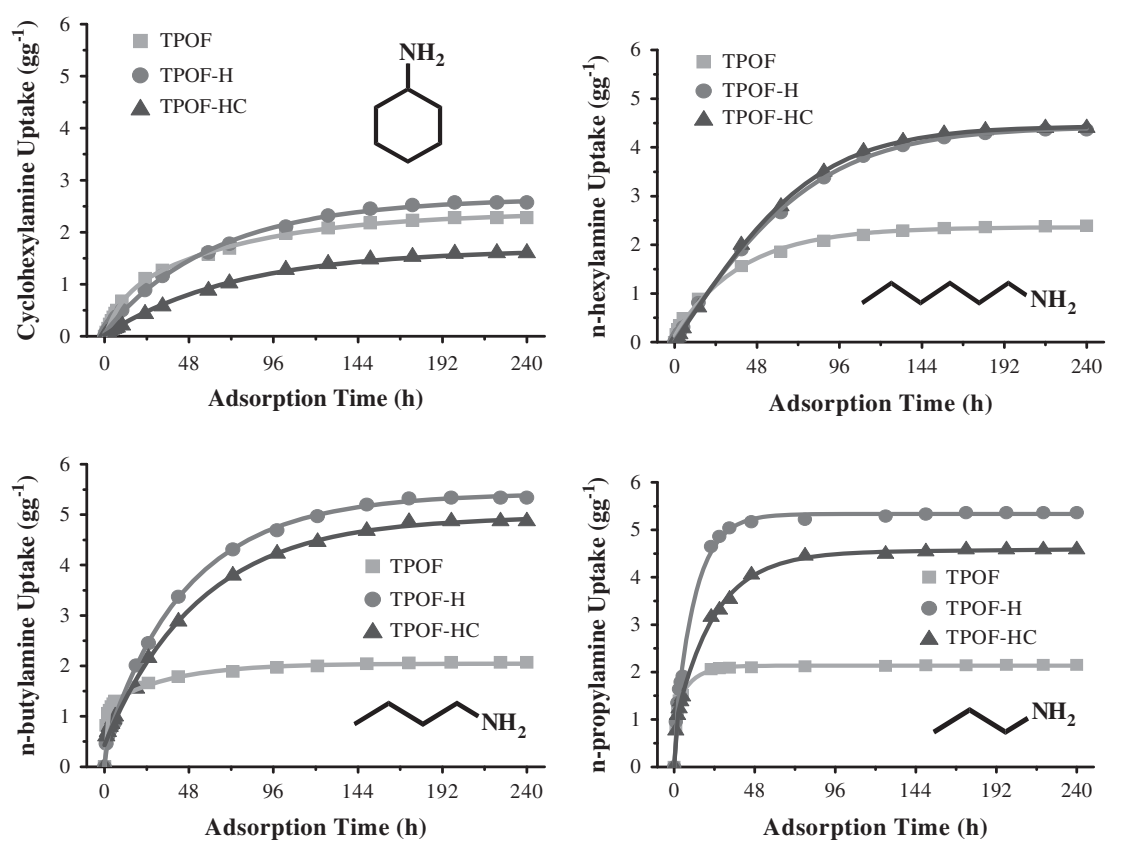

Figure 5 Vapor uptakes of cyclohexylamine, $n$-hexylamine, $n$-butylamine and $n$-propylamine by TPOF, TPOF-H and TPOF-HC. TPOF, triptycene-based porous organic framework; TPOF-H, TPOF with hydroxyl groups; TPOF-HC, TPOF with hydroxyl and carboxyl groups. A full color version of this figure is available at the Polymer Journal online.

for linear amines over $n$-hexane: the adsorption selectivities of TPOF-H for $n$-propylamine, $n$-butylamine and $n$-hexylamine over $n$-hexane are 26.8, 26.7 and 21.8, respectively, whereas for TPOF-HC, they are as high as $32.7,34.8$ and 31.4 , respectively. Such a combination of high vapor capacity and selectivity for VOC adsorption has rarely been reported in the literature for microporous polymers. ${ }^{14}$

The POFs do not dissolve in any organic solvents, suggesting hyper-crosslinked network structures. The thermogravimetric analysis revealed that all of the POFs exhibit high decomposition temperatures 

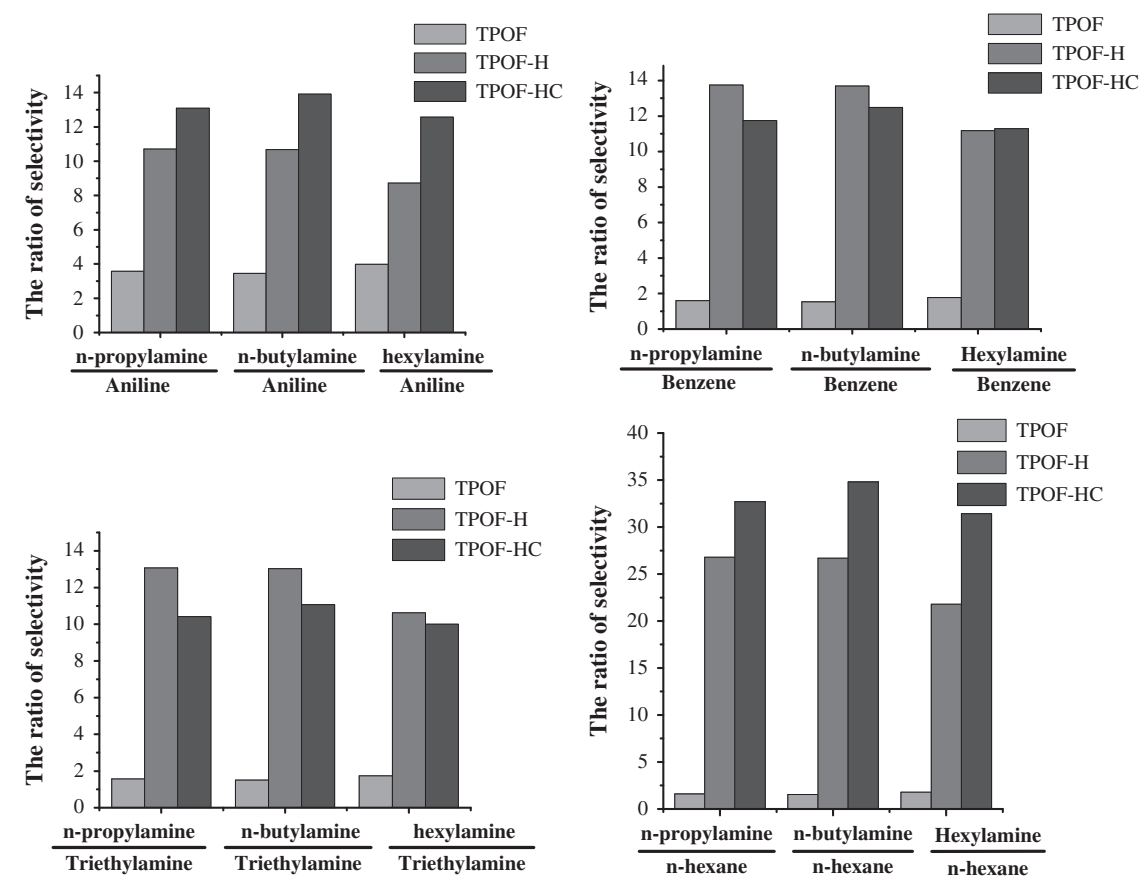

Figure 6 Adsorption selectivity for aliphatic amines over aniline, benzene or triethylamine by TPOF, TPOF-H and TPOF-HC. TPOF, triptycene-based porous organic framework; TPOF-H, TPOF with hydroxyl groups; TPOF-HC, TPOF with hydroxyl and carboxyl groups. A full color version of this figure is available at the Polymer Journal online.
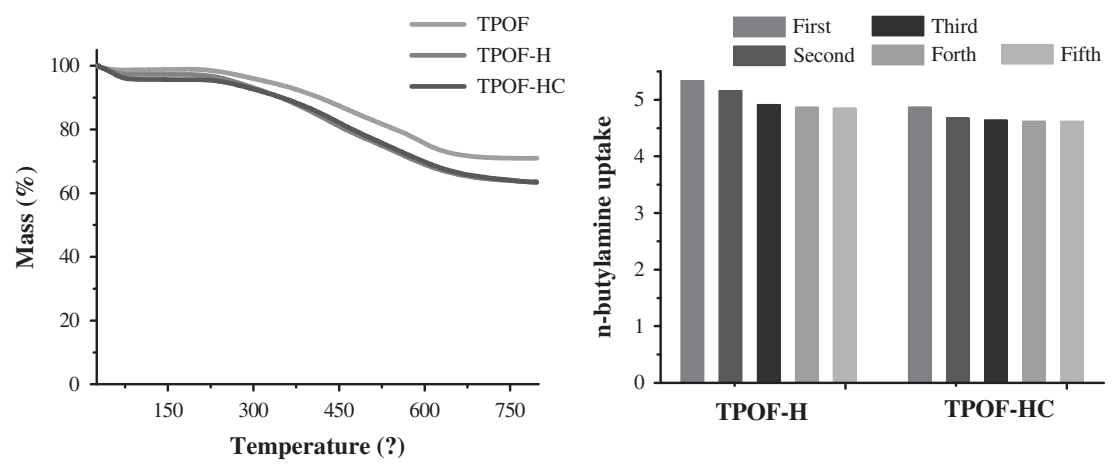

Figure 7 Thermogravimetric analysis (TGA) curves and the recyclability of TPOF, TPOF-H and TPOF-HC. TPOF, triptycene-based porous organic framework; TPOF-H, TPOF with hydroxyl groups; TPOF-HC, TPOF with hydroxyl and carboxyl groups. A full color version of this figure is available at the Polymer Journal online.

above $400{ }^{\circ} \mathrm{C}$ and char yields of more than $60 \mathrm{wt} \%$ at $800{ }^{\circ} \mathrm{C}$ (Figure 7). Because of their high chemical and thermal stabilities, there were no observable deteriorations of their absorption capabilities for VOC vapors after recycling 5 times.

\section{CONCLUSIONS}

TPOFs with high BET surface areas and tailored pore environments were designed and synthesized through a facile external knitting strategy. Compared with unmodified TPOF, TPOF-H and TPOF-HC functionalized with hydroxyl and/or carboxyl groups exhibit very high aliphatic amine vapor uptake capacities of up to 5 times their own weight and high selectivities of more than 30 times for aliphatic amines over $n$-hexane. This extremely high vapor uptake and selectivity should be because of a synergistic effect between high porosity and high hydrogen bonding functionalized pore microenvironments. The research revealed that the microporous environment is also of vital importance in the design of nextgeneration adsorption materials. The VOCs can be desorbed from the POFs by heating the samples under vacuum. Such a unique combination of high adsorption capability, selectivity and facile synthesis would open up new opportunities for their use in environment-related applications.

\section{CONFLICT OF INTEREST}

The authors declare no conflict of interest.

\section{ACKNOWLEDGEMENTS}

Financial support from the National Natural Science Foundation of China (20974069 and 21174089) and the State Key Laboratory of 
Molecular Engineering of Polymers (Fudan University) (no. K2013-23) is acknowledged.

1 Manjare, S. D. \& Ghoshal, A. K. Studies on adsorption of ethyl acetate vapor on activated carbon. Ind. Eng. Chem. Res. 45, 6563 (2006).

2 Amari, A., Chlendi, M., Gannouni, A \& Bellagi, A. Experimental and theoretical studies of VOC adsorption on acid-activated bentonite in a fixed-bed adsorber. Ind. Eng. Chem. Res. 49, 11587 (2010).

3 Guillemot, M., Mijoin, J., Mignard, S. \& Magnoux, P. Adsorption of tetrachloroethylene on cationic $X$ and $Y$ zeolites: influence of cation nature and of water vapor. Ind. Eng. Chem. Res. 46, 4614 (2007).

4 Serrano, D. P., Calleja, G., Botas, J. A. \& Gutierrez, F. J. Adsorption and hydrophobic properties of mesostructured MCM-41 and SBA-15 materials for volatile organic compound removal. Ind. Eng. Chem. Res. 43, 7010 (2004).

$5 \mathrm{Hu}$, X. J., Qiao, S. Z., Zhao, X. S. \& Lu, G. Q. Adsorption study of benzene in ink-bottle-like MCM-41. Ind. Eng. Chem. Res. 40, 862 (2001).

6 Stenzel, M. H. Remove organics by activated carbon adsorption. Chem. Eng. Progr. 3 89, 36 (1993).

7 Khan, F. I. \& Kr Ghoshal, A. Removal of volatile organic compounds from polluted air. J. Loss Prevention Proc. Ind. 13, 527 (2000).

8 Wang, J., Wang, G., Wang, W., Zhang, Z., Liu, Z. \& Hao, Z. Hydrophobic conjugated microporous polymer as a novel adsorbent for removal of volatile organic compounds. J. Mater. Chem. A 2, 14028 (2014).

9 Chen, L., Yang, Y., Guo, Z. \& Jiang, D. Highly efficient activation of molecular oxygen with nanoporous metalloporphyrin frameworks in heterogeneous systems. Adv. Mater. 23, 3149 (2011)
$10 \mathrm{Xu}$, H., Chen, X., Gao, J., Lin, J., Addicoat, M., Irle, S. \& Jiang, D. Catalytic covalent organic frameworks via pore surface engineering. Chem. Commun. 50, 1292 (2014).

$11 \mathrm{Wu}$, K., Guo, J. \& Wang, C. Dispersible and discrete metalloporphyrin-based CMP nanoparticles enabling colorimetric detection and quantitation of gaseous SO2. Chem. Commun. 50, 695 (2014).

12 Sigen, A., Zhang, Y., Li, Z., Xia, H., Xue, M., Liu, X. \& Mu, Y. Highly efficient and reversible iodine capture using a metalloporphyrin-based conjugated microporous polymer. Chem. Commun. 50, 8495 (2014).

13 Liu, X., Sigen, A., Zhang, Y., Luo, X., Xia, H., Li, H. \& Mu, Y. A porphyrin-linked conjugated microporous polymer with selective carbon dioxide adsorption and heterogeneous organocatalytic performances. RSC. Adv. 4, 6447 (2014).

14 Liu, X., Xu, Y., Guo, Z., Nagaia, A. \& Jiang, D. Super absorbent conjugated microporous polymers: a synergistic structural effect on the exceptional uptake of amines. Chem. Commun. 49, 3233 (2013).

15 Chen, C. \& Ma, Y. Iptycenes Chemistry: From Synthesis to Applications, (Springer-Verlag, Berlin Heidelberg, 2013).

16 Chong, J. H. \& MacLachlan, M. J. Iptycenes in supramolecular and materials chemistry. Chem. Soc. Rev. 38, 3301 (2009).

17 Crane, A. K., Wong, E. Y. L. \& MacLachlan, M. J. Metal-organic frameworks from novel flexible triptycene- and pentiptycene-based ligands. CrystEngComm. 15, 9811 (2013).

18 Zhang, C., Wang, Z., Wang, J., Tan, L., Liu, J., Tan, B., Yang, X. \& Xu, H. Synthesis and properties of triptycene-based microporous polymers. Polymer 54, 6942 (2013).

19 Mckeown, N. B., Budd, P. M. \& Book, D. Microporous polymers as potential hydrogen storage materials. Macromol. Rapid Commun. 28, 995 (2007).

20 Luo, Y., Li, B., Wang, W., Wu, K. \& Tan, B. Hypercrosslinked aromatic heterocyclic microporous polymers: a new class of highly selective $\mathrm{CO} 2$ capturing materials. Adv. Mater. 24, 5703 (2012)

21 Li, B., Gong, R., Wang, W., Huang, X., Zhang, W., Li, H., Hu, C. \& Tan, B. A new strategy to microporous polymers: knitting rigid aromatic building blocks by external cross-linker. Macromolecules 44, 2410 (2011). 